\title{
Birth companion of choice: a survey amongst women who have given birth at a rural hospital in Limpopo, South Africa
}

\begin{abstract}
Background: Advancements in medical interventions have contributed to the prevention of innumerable deaths of women and newborns globally, leading to the medicalization of childbirth, which may have inadvertently contributed to a dehumanization of the birthing experience by denying women the social and emotional support that they enjoyed when giving birth in the home environment. The WHO places equal importance on the quality of clinical care and the quality of experience of care. Emotional support, provided by a birth companion of choice, is critical for enhancing the experience of care during labour and birth. This study explores advocacy for women to have a birth companion, as well as perceptions of women about having a birth companion during labour and birth in a rural sub-district of Limpopo Province in South Africa.
\end{abstract}

Methods: A cross-sectional study based on a continuous electronic RMC client satisfaction survey, on an on-line platform, which was completed by 506 women in a postnatal ward of a rural district hospital. Observations of interactions between midwives, women in labour and their birth companions during labour and birth were conducted in the same hospital. The survey data was analysed using descriptive statistics in Excel, and comprises both univariate and bi-variate analyses. The qualitative data from the observations was analysed using thematic analysis techniques.

Results: Over $80 \%$ of women who completed the survey reported that they were informed about what a birth companion is and encouraged to register a birth companion at the health facility where they were attending antenatal care. Almost half of the women (49\%) who were encouraged to bring a birth companion, had a birth companion during labour, whereas $15 \%$ of those not encouraged to bring a birth companion, had a birth companion during labour. The respondents who were feeling neutral, happy and very happy about having a birth companion were most likely to recommend having a birth companion to their family member or friends.

Conclusion: The findings show that encouraging and supporting the presence of a woman's companion of choice during labour and childbirth is an effective intervention that is respectful of women's autonomy and agency and which is crucial for improving quality of clinical care and the experience of care during labour and birth.

Keywords: birth companion, quality of care, experience of care, labour and birth, Limpopo
Volume 6 Issue 6 - 2020

\author{
Joy $\vee$ Summerton, Tsakani Mtileni \\ Department of Obstetrics and Gynaecology, University of \\ Limpopo/University of Limpopo Trust, South Africa
}

\begin{abstract}
Correspondence: Joy $\vee$ Summerton, Department of Obstetrics and Gynaecology, University of Limpopo/University of Limpopo Trust, C/O 7I Tzaneen Road and University Street, Mankweng Township, Polokwane, Limpopo Province, 0727, South Africa, Email joy.summerton@ul.ac.za
\end{abstract}

Received: November 03, 2020 | Published: November 20, 2020

\begin{abstract}
Abbreviations: ANC,
antenatal care; IEC, information education and communication; RMC, respectful maternity care; WHO, World health organisation
\end{abstract}

\section{Background}

There is a global interest in improving the quality of maternal and newborn care through initiating, supporting and sustaining programmes to this end. Over the past few decades the global focus was predominantly on improving the quality of clinical care, thus advocating for health facility-based delivery. This has contributed immensely to the prevention of innumerable deaths of women and newborns globally. Consequently it has led to the medicalization of childbirth, which may have unintentionally contributed to a dehumanization of the birthing experience by denying women the social and emotional support that they enjoyed when giving birth in the home environment. ${ }^{1-3}$ This realization has evoked a consciousness to afford equal emphasis and importance to improving the quality of experience of care as an essential element of improving health outcomes, as well as to humanize the birthing experience. ${ }^{4}$ The World Health Organisation (WHO) has played a pivotal role in advocating for the positioning of quality of experience of care on an equal level with the provision of clinical care.

The WHO defines quality of care as ${ }^{5}$ the provision of technically competent care, which includes evidence-based practices for routine care and the management of complications, as well as actionable health management information systems and functional referral systems; and the enhancement of women's experience of care, which includes informative and comprehensible communication, care delivered with respect and for women's dignity, choices and autonomy in decisionmaking, and availability of social, emotional and practical support.

The WHO definition of quality of care during childbirth in health facilities reflects both the provision of care and the experience of care, within the available physical infrastructure, supplies, management and human resources with the knowledge, skills and capacity to 
manage pregnancy and childbirth. ${ }^{6}$ A number of policy directives and guidelines have been issued by the WHO which explicitly and intrinsically link clinical care and experience of care. ${ }^{7-9}$ Respectful Maternity Care (RMC) has been unequivocally delineated as an essential component of high-quality care in both the WHO recommendations for intrapartum care for a positive childbirth experience, as well as the WHO standards for improving the quality of maternal and newborn care in health facilities. ${ }^{10,11}$ The quality of standards framework identifies 3 domains under the experience of care: effective communication, respect and preservation of dignity, and emotional support. Emotional support includes ensuring that every woman: is given the option to have a companion of choice during labour and birth; is allowed to be ambulatory during the first stages of labour; is permitted to take fluids and food during the early stages of labour; and is encouraged to give birth in the position of the woman's choice.

Birth companionship is a key component of providing RMC. In this paper, birth companionship refers to the provision of any type of support provided by a lay companion at any time during the intrapartum period (labour and birth). There is a dearth of evidence on the benefits of a birth companion during labour and birth. Birth companionship is associated with enhanced positive experience of care, as well as positive health outcomes for both women and newborns. Various studies, including two Cochrane reviews of continuous support and birth companionship, have found that women who receive continuous support during labour were more likely to have shorter labour and spontaneous vaginal birth, decreased usage of intrapartum anaelgesia, caesarian section and episiotomies, and their babies were less likely to have low five-minute Apgar scores (less than 7). ${ }^{12-16}$ Not entirely incomprehensible if one considers that childbirth naturally triggers fear for many women. Fear, exacerbated by isolation from family members in a labour ward, together with the anxiety of being in a strange and unfamiliar environment and often negative attitudes from health care providers, can alter the physiology of labour by increasing substances that inhibit the synthesis of oxytocin and endogenous endorphins that are essential for cervical effacement and dilatation. ${ }^{17,18}$ It is also reported that excessive anxiety increases an endogenous release of catecholamines, which have the effect of reducing blood flow to and from the placenta, and can thus restrict foetal oxygen availability and waste removal, as well as reduced effectiveness of uterine contractions (powers), which inevitably slows labour progression. ${ }^{19,20}$

Furthermore, women who have continuous support during labour are generally more satisfied with the experience of labour and birth, are more satisfied with health care services provided, and some studies even suggest that women who have a birth companion are less likely to experience mistreatment during childbirth. ${ }^{21-24} \mathrm{~A}$ study in Iran found that continuous support during labour also decreased the incidence of postpartum depression. ${ }^{25-27}$

Despite the overwhelming evidence of the benefits of continuous support by birth companions during labour and birth for improving the quality of care and experience of labour and birth, implementation of this approach is not universal. Birth companionship is increasingly being included in the maternal health guidelines of many countries, such as Kenya and South Africa. The National Guidelines for Quality Obstetrics and Perinatal Care in Kenya recommends that health care providers encourage women to have their birth companion of choice with them during the first and second stages of labour, and that facilities should make arrangements to accommodate birth companions. ${ }^{28,29}$ The South African Guidelines for Maternal Care states that family and friends should be allowed to provide birth companionship during labour. ${ }^{30}$ Furthermore, the new South African Maternity Case Record requires health care providers to inform pregnant women about their right to have a birth companion of choice and to record that such information was shared with women.

There remains inadequate knowledge and evidence on the extent to which birth companionship is actually practiced in South Africa, and more specifically in the public health sector in Limpopo. A study in a tertiary hospital in Limpopo revealed that none of the midwives in the study encouraged the presence of a birth companion during labour and birth. ${ }^{31}$ The Limpopo Department of Health undertook to pilot a RMC model in a sub-district of Limpopo which had consistently high rates of maternal mortality and perinatal mortality due to birth asphyxia. The pilot entailed mentoring and supporting midwives and support staff at 22 health care facilities to implement RMC. All women attending antenatal care at the facilities were informed about birth companionship and the benefits thereof. A birth companion pamphlet with key information about the role and responsibilities of a birth companion was designed and translated into the predominant language in the sub-district (Sepedi), to compliment the verbal information shared with both pregnant women and birth companions during antenatal care visits. Women were requested to identify a birth companion that they trust and feel confident could fulfil the articulated roles and responsibilities. The birth companions were registered in a facility-based birth companion registration book and were required to accompany pregnant women for at least one antenatal care visit in order to acquire the necessary knowledge and skills to provide effective support during labour and birth. This paper aims to enhance insights into the promotion of birth companionship by health care providers in the pilot site, as well as the perceptions of women about having a birth companionship during labour and birth.

\section{Methods}

\section{Design}

The study is a cross-sectional study based on a continuous electronic RMC client satisfaction survey, on an on-line platform which has been developed to capture the perceptions of women about the respectful care provided to them during labour and birth. The survey is implemented in the only district hospital, in a sub-district in Limpopo. The survey has been placed on tablets that are mounted in strategic and accessible places in the postnatal ward. Women were encouraged by an independent survey administrator to complete the survey as soon as possible after birth when they have arrived in the postnatal ward. Women who have delivered by caesarian section were provided with a roving tablet where they were able to complete the survey in the comfort of their bed. The survey consists of 17 closed ended questions and 1 open ended question, and takes an average of 10 minutes to complete. Once a participant has completed the survey, responses are sent directly to a central repository, and cannot be retrieved or accessed on the tablets at the hospital. This security feature has had a positive effect in encouraging participation in the survey at the hospital, as many clients initially feared that their responses would be retrieved by hospital staff. The survey is entirely anonymous and responses cannot be traced back to a specific respondent. Participants have the option of completing the survey in either English or the local vernacular, Sepedi.

The survey questions are designed to assess some of the key components of the RMC initiative, as perceived by clients, namely communication, information, pain relief and emotional support as outlined in the WHO recommended Quality of Care Framework. 


\section{Sampling and data collection}

During the period August 2019 and March 2020, 506 participants, out of a total of 3197 women who delivered at the facility, completed the survey after giving birth. All women were verbally encouraged to complete the survey by an independent survey administrator stationed at the postnatal ward of the maternity unit. Although the administrator only works during the day shift, she is still able to interact with clients that deliver during the night the following day as no clients are discharged during the night. The introduction section in the survey prompts participants to proceed to Question 1 if they consent to participate in the survey. If they do not consent, they have the option to exit the survey. The survey also clearly stated that participation was voluntary and that participants could discontinue their participation at any stage of the survey. All 506 participants who completed the survey over the 8 -month period were included in the study.

\section{Data analysis}

The survey data was exported from SoGoSurvey using the predefined reports (which excluded incomplete records) into Microsoft Excel (Excel) format. The data was coded to form 4 twomonth time intervals to ascertain changes over time. The data from the two language versions were then merged to form 1 database. The data was analysed using descriptive statistics in Excel, and comprises both univariate and bi-variate analyses.

\section{Results}

This paper only presents the data related to birth companionship, from the survey.

\section{Informed about birth companionship}

The majority of women who gave birth at the pilot hospital attended antenatal care (ANC) at a primary health care facility within the sub-district in which RMC was piloted. Only women who have been assessed as highrisk attend ANC at the hospital, as per policy. Due to close proximity and/or personal preference, some women from sub-districts outside of the pilot sub-district also give birth at the pilot hospital. Some women reside outside of the province, where they attend ANC, but return to the sub-district to give birth at the pilot hospital (Figures 1-3).

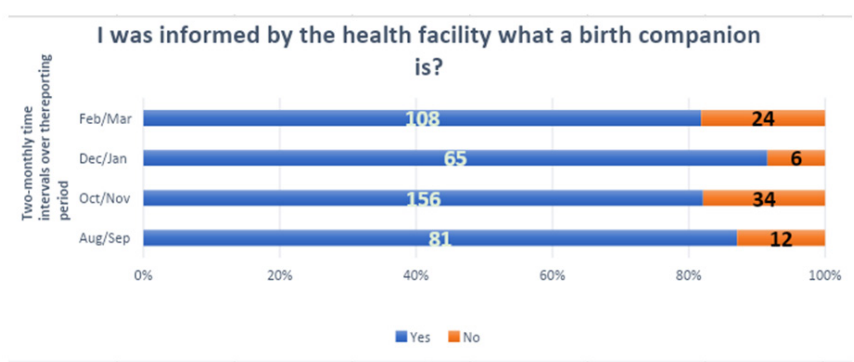

Figure I Informed about a birth companion.

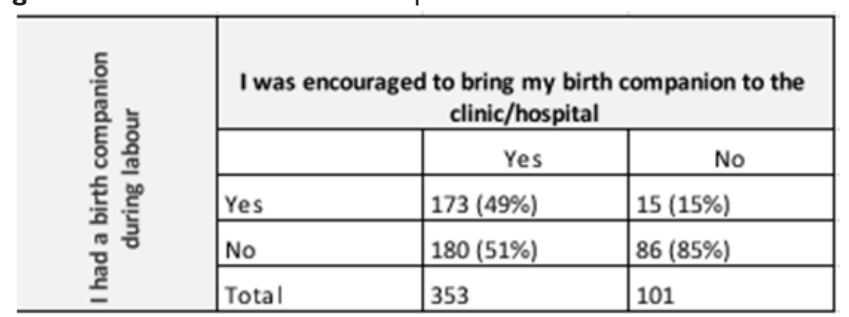

Figure 2 Encouraged to bring a birth companion vs having a birth companion.

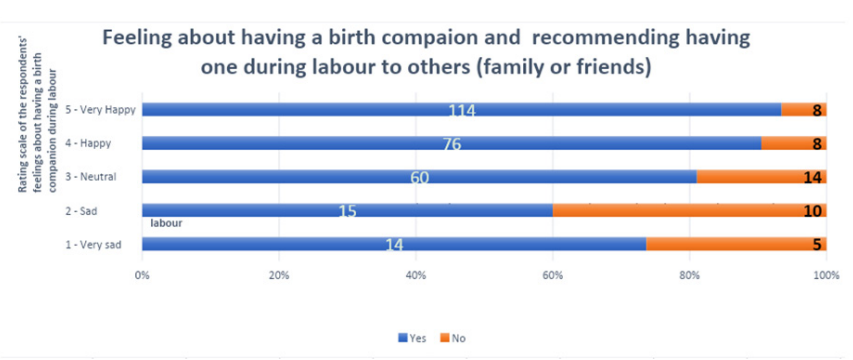

Figure 3 Feelings about a birth companion vs recommending a birth companion.

Over $80 \%$ of women who completed the survey reported that they were informed about what a birth companion is at the health facility where they were attending ANC. All the women who were informed were from a health facility within the pilot sub-district. None of the women who attended ANC outside of the pilot sub-district were informed about what a birth companion is at their health facility during ANC.

Over $80 \%$ of women were informed about what a birth companion is at their health facility during ANC, they were also encouraged to identify and register a birth companion of choice to accompany and support them during labour and birth. Each primary health care facility in the pilot sub-district has developed a birth companion register where birth companions chosen by pregnant women are registered by the health facility and provided with information on the roles and responsibilities of a birth companion during labour and birth.

Almost half of the women (49\%) who were encouraged to bring a birth companion during $\mathrm{ANC}$, had a birth companion during labour, whereas $15 \%$ of those not encouraged to bring a birth companion, had a birth companion during labour. Not being encouraged to bring a birth companion during antenatal care was directly linked to not having a birth companion during labour and birth. The respondents who were feeling neutral, happy and very happy about having a birth companion were most likely to recommend having a birth companion to their family member or friends. It is notable that some respondents, even if a very small proportion, who were unhappy and even very unhappy, reported that they would still recommend having a birth companion to other women. Similarly, an equally small proportion of women who reported being happy and even very happy with having a birth companion reported that they would not recommend a birth companion to other women.

\section{Discussion}

The survey and observations cover a much broader scope of respectful maternity care. For the purpose of this paper, only the findings related to birth companionship are presented. The findings of the survey indicate that the health facilities in the RMC pilot subdistrict are informing pregnant women who attend ANC about birth companionship. Although the exact nature and manner in which the information about birth companionship is shared is unknown. One of the components of the RMC pilot is antenatal education classes to prepare pregnant women and their birth companions for the pregnancy, labour and birth journey (what to expect and what to do). The classes are facilitated by a midwife, supported by an interdisciplinary team, and guided by an antenatal education classes facilitator manual and toolkit comprising of teaching and learning aids (posters, pelvic model to simulate the birthing process, etc.), as well as birth companion tools to demonstrate non-pharmacological pain relief techniques (birthing ball, massages, cold and heat packs, 
etc.). Posters of the benefits of having a birth companion have also been mounted at strategic places within the health facilities in the RMC pilot-district. The initial introduction of the birth companion concept was vehemently opposed by health care providers who cited i) lack of space in the labour ward, ii) lack of uptake by community members who felt childbirth should not be observed by lay persons; iii) interference of birth companions with the clinical duties and responsibilities of health care providers; iv) inappropriate behavior of birth companions that may infringe on the privacy of other women in labour (e.g. taking photos); and v) breaching patient/provider confidentiality (birth companions being privy to clinical information about the woman in labour), as some of the major barriers to affording women the right to be supported by a birth companion during labour and birth. The perceived barriers amongst health care providers to birth companionship are also reported in other studies both in South Africa and other developing countries. ${ }^{32-35}$

The findings of the study also show that a small proportion of women who were informed about birth companions at their health facility where they were attending ANC, did not have a birth companion during labour and birth. The survey did not ask women to explain why they did not have a birth companion, which was a significant limitation in the design of the survey. There are a number of reasons which could be attributed to the absence of a birth companion for this strata of women. For one, there are studies where women have reported not to desire a birth companion during labour and birth. ${ }^{36}$ Another reason could be that the benefits of a birth companion were not adequately presented to pregnant women during ANC or antenatal education classes, or that the birth companion was not available at the onset of labour. The latter reason was provided by some women during the observations. For to the RMC pilot, it was recommended that health care providers request women to identify a reserve birth companion during ANC visits, in the event that a registered birth companion is not available at the onset of labour. It is then incumbent on the health care provider to capacitate the reserve birth companion to provide effective emotional and physical support to the woman in labour upon their arrival at the labour ward. Based on observations, this was not generally initiated by health care providers, unless prompted to do so by the RMC mentors, when they are at the facility. This concurs with the findings of one other study conducted at a hospital in Limpopo, which reported that no midwife in the study encouraged the presence of a companion during childbirth. ${ }^{37}$ The observations revealed that in the instances where health care providers supported women to identify a reserve birth companion, the majority of birth companions were available and willing, and provided support that was appreciated by the woman in labour once the birth companion was capacitated to do so.

According to the findings of the survey, women who were not informed about a birth companion did not attend ANC at facilities within the pilot sub-district and were thus not required to identify and register a birth companion, nor were they exposed to antenatal education classes for pregnant women and their birth companions and were most likely not privy to target specific Information Education and Communication (IEC) material on birth companionship. It is prudent to note that these are critical advocacy tools and strategies to normalize birth companionship, within a system with underlying malaise, at best, and outright animosity at worst, towards birth companions in the labour ward.

In spite of women not being informed about birth companions during ANC or any other interaction with the health care system, some women $(15 \%)$ still had a birth companion during labour and birth. This is encouraging and once again affirms the innate desire for women to have the presence of someone familiar and that they trust during labour, and who is available for them throughout labour and birth, as highlighted in numerous studies. ${ }^{38-40}$ It is especially important for health care providers to discern a women's need and clinical benefit for continuous support throughout labour versus health care providers' availability to provide continuous support to every woman in labour. With the progression of the RMC implementation in the pilot sub-district, health care providers were increasingly appreciating the presence of birth companions. This was largely attributed to the perceived usefulness of the birth companions in facilities with a dire shortage of nurses and midwives. This finding is consistent with studies in other countries where companions are considered a low cost intervention that is valued in facilities with shortage of nursing staff. ${ }^{41}$

It is of concern that a small proportion of women who had a birth companion were not happy with the experience and similarly, a small proportion of woman who reported being happy with the experience of having a birth companion would not recommend it to another woman. There is a possibility that the questions were not assimilated by some respondents and thus provided invalid responses. However, where respondents had assimilated the questions, reasons for their responses would provide critical input to explore convergent and divergent perspectives on women's needs.

\section{Limitations}

The study has provided valuable insights on the extent to which birth companionship has been advocated for and integrated into the health care system in the pilot sub-district. However, it has a number of limitations. The study did not assess the nature of support provided by the birth companions, as this could have enriched the findings on whether or not a birth companion would be recommended to other women. The study also did not collect data about the type of birth companion, which could also have enhanced insights into which type of birth companion is preferred by women. In addition, the findings of the study could have further enriched insights into birth companionship had it explored the reasons for women who were encouraged to bring a birth companion during labour, but did not have a birth companion during labour. Similarly, there is a need to further explore the reasons for women who were happy about having a birth companion yet would not recommend having a birth companion to other women, as well as women who were not happy with having a birth companion but would recommend it to other women.

\section{Conclusion}

Encouraging and supporting the presence of a woman's companion of choice during labour and childbirth is an effective intervention that is respectful of women's autonomy and agency and which is crucial for improving quality of care during labour and birth. It should be recognized that introducing labour companionship is complex and requires a reorganization and restructuring of services, underpinned with changing health care provider attitudes and behavior. The RMC pilot used strategies such as sensitization activities and sharing evidence-based information with health care providers, creating safe spaces for health care providers to express their reservations and concerns about birth companionship, supporting health care providers to address system barriers such as privacy and space, and sharing women's positive experiences with having a birth companion with health care providers. Policies and guidelines alone do not equate to quality of care nor the humanization of childbirth. There is an urgent need to prioritise bridging the divide between proclamations and practice surrounding birth companionship within the context of human rights, patient centred-care and respectful care. The time to 
acknowledge and reiterate the need and benefits of continuous support during labour and birth has long passed. It needs to be deliberately and unapologetically actioned.

\section{Declaration}

\section{Ethics approval and consent to participate}

Ethics approval was obtained from the University of Limpopo Turfloop Research Ethics Committee (TREC/59/2019:IR). Administrative approval was obtained from the Limpopo Department of Health managers, at provincial, district, sub-district and facility levels prior to data collection (survey going live). Informed consent is built into the online survey, which also explains to participants that participation in the survey is voluntary and that they may discontinue at any point during the survey. Thus consent was obtained from all participants who participated in the survey. Confidentiality, privacy and anonymity of participants were maintained at all times. The survey does not request any identifying information (name, address, identification number, contact details) of participants, and once participants submit their responses, they are sent to a repository that is password protected, and no longer retrievable on the survey devices.

\section{Consent for publication}

Both authors, JVS and TRM, hereby give consent for the manuscript to be published.

\section{Availability of data and materials}

The datasets and observation notes will be made available upon a valid request to the corresponding author (Joy Summerton, joy. summerton@ul.ac.za).

\section{Acknowledgements}

The authors and principal researchers wish to convey their humble gratitude to the Limpopo Department of Health maternal and newborn care programmes, managers from the district and pilot sub-district, as well as frontline health care providers from the district and pilot sub-district for being true advocates for respectful and patient-centred care.

\section{Conflicts of interest}

The authors declare that they have no competing interests.

\section{Authors contributions}

JVS and TRM contributed equally to the conceptualization, implementation, data analysis and writing of the manuscript. Both authors read and approved the manuscript. The questionnaire that was used in the study was specifically developed for this study.

\section{Funding}

The study was co-funded by the Discovery Foundation, the Discovery Fund and UNICEF. The funding organisations did not have any role in the design of the study, data collection, analysis, interpretation of data, and writing the manuscript.

\section{References}

1. Perkins J, Rahman AE, Mhajabin S, et al. Humanised childbirth: the status of emotional support of women in rural Bangladesh. Sexual and Reproductive Health Matters. 2019;27(1):228-247.

2. Johnson R, Newburn M, Macfarlane A. Has the medicalization of childbirth gone too far? British Medical Journal (BMJ). 2002;24(7342):892-895.
3. Brubaker SJ, Dilaway HE. Medicalization, natural childbirth and birthing experiences. Sociol Compass. 2009;3(1):31-48.

4. Tunçalp Ö, WereWM, MacLennan C, et al. Quality of care for pregnant women and newborns - the WHO vision. BJOG. 2015;122(8):10451049 .

5. Ojelade OA, Titiloye MS, Bohren MA, et al. The communication and emotional support needs to improve women's experience of childbirth care in health facilities in Southwest Nigeria: a qualitative study. International Journal of Gynecology and Obstetrics. 2017;139(1):2737.

6. World Health Organisation. WHO recommendations for augmentation of labour. Geneva: World Health Organisation; 2014.

7. World Health Organisation. WHO recommendations on antenatal care for a positive pregnancy experience. Geneva: World Health Organisation; 2016.

8. World Health Organisation. WHO recommended interventions for improving maternal and newborn health: integrated management of pregnancy and childbirth. Geneva: World Health Organisation; 2007.

9. World Health Organisation. Standards for improving quality of maternal and newborn care in health facilities. Geneva: World Health Organisation; 2016.

10. World Health Organisation. WHO recommendations: intrapartum care for a positive childbirth experience. Geneva: World Health Organisation; 2018.

11. World Health Organisation. Companion of choice during labour and childbirth for improved quality of care. Geneva: World Health Organisation; 2016.

12. Afulani P, Kusi C, Kirumbi L, et al. Companionship during facilitybased childbirth: results from a mixed-methods study with recently delivered women and providers in Kenya. BMC Pregnancy and Childbirth. 2018;18(150).

13. Bohren MA, Hofmeyr GJ, Sakala C, et al. Continuous support for women during childbirth (Review). Cochrane Database of Systematic Reviews. 2017;7:CD003766.

14. Bohren MA, Berger BO, Munthe-Kaas H, et al. Perceptions and experiences of labour companionship: a qualitative evidence synthesis (Review). Cochrane Database of Systematic Reviews. 2019;3:CD012449.

15. Hodnett ED, Gates S, Hofmeyer GJ, et al. Continuous support for women during childbirth. Cochrane Library Protocols. 2013;12.

16. Souza SRRK, Gualda DMR. The experience of women and their coaches with childbirth in a public maternity hospital. Texto \& Contexto Enfermagem. 2016;25(1):1-9.

17. Santos LM, Barbosa TS, Paiva MS, et al. Postpartum women's perception on the coach's participation during the delivery process. JNUOL. 2011;5(5).

18. Yuenyong S, Jirapaet V, O'Brien B. Support from a Close Female Relative in Labour: The Ideal Maternity Nursing Intervention in Thailand. J Med Assoc Thai. 2008;91(2):253-260.

19. Thompson ED. Maternal and pediatric nursing. Philadelphia: WB Saunders. 1995.

20. Afulani P, Kusi C, Kirumbi L, et al. ompanionship during facility-based childbirth: results from a mixed-methods study with recently delivered women and providers in Kenya. BMC Pregnancy and Childbirth. 2018;18(150).

21. Diamond-Smith N, Sudhinaraset M, Melo J. The relationship between women's experiences of mistreatment at facilities during childbirth, types of support received and person providing the support in Lucknow, India. Midwifery. 2016; 40:114-123. 
22. Abuya T, Warren CE, Miller N, Njuki R, Ndwiga C, Maranga A, et.al. Exploring the prevalence of disrespect and abuse during childbirth in Kenya. PLoS One. 2015;10.

23. Souza SRRK, Gualda DMR. The experience of women and their coaches with childbirth in a public maternity hospital. Texto \& Contexto Enfermagem. 2016;25(1):1-9.

24. Fathi Najafi T, Latifnejad Roudsari R, Ebrahimipour H. The best encouraging persons in labour: A content analysis of Iranian mothers' experiences of labour support. PLoS ONE. 2017;12(7):e0179702.

25. Hassazahraee R, Fahami F, Yazdani M, et al. Supportive role of the midwife in preventing of postpartum depression. The J Qazvin Uni of Med Sci. 2003;5:19-26.

26. Masoudnia E. Relationship between Perceived Social Support and Risk of Postpartum Depression Disorder. IJNR. 2012;70(24):8-18.

27. Afulani P, Kusi C, Kirumbi L, et al. Companionship during facilitybased childbirth: results from a mixed-methods study with recently delivered women and providers in Kenya. BMC Pregnancy and Childbirth. 2018;18(150).

28. Minister of Public Health and Sanitation and Ministry of Medical Services. National Guidelines for quality obstetrics and perinatal care, Kenya; 2012.

29. Department of Health. Guidelines for Maternity Care in South Africa. 4th ed. 2016.

30. Maputle MS. Support provided by midwives to women during labour in a public hospital, Limpopo Province, South Africa: a participant observation study. BMC Pregnancy and Childbirth. 2018;12(210).

31. Souza SRRK, Gualda DMR. The experience of women and their coaches with childbirth in a public maternity hospital. Texto \& Contexto Enfermagem. 2016;25(1):1-9.

32. Kabakian-Khasholian T, Portela A. Companion of choice at birth: factors affecting implementation. BMC Pregnancy and Child Health. $2017 ; 17(265)$.
33. Bohren MA, Berger BO, Munthe-Kaas H, et al. Perceptions and experiences of labour companionship: a qualitative evidence synthesis (Review). Cochrane Database of Systematic Reviews. 2019;3:CD012449.

34. Afulani P, Kusi C, Kirumbi L, et al. Companionship during facilitybased childbirth: results from a mixed-methods study with recently delivered women and providers in Kenya. BMC Pregnancy and Childbirth. 2018;18(150).

35. Maputle MS. Support provided by midwives to women during labour in a public hospital, Limpopo Province, South Africa: a participant observation study. BMC Pregnancy and Childbirth. 2018;12(210).

36. Ojelade OA, Titiloye MS, Bohren MA, et al. The communication and emotional support needs to improve women's experience of childbirth care in health facilities in Southwest Nigeria: a qualitative study. International Journal of Gynecology and Obstetrics. 2017;139(1):2737.

37. Bohren MA, Berger BO, Munthe-Kaas, et al. Perceptions and experiences of labour companionship: a qualitative evidence synthesis (Review). Cochrane Database of Systematic Reviews. 2019;3:CD012449.

38. Kabakian-Khasholian T, Portela A. Companion of choice at birth: factors affecting implementation. BMC Pregnancy and Child Health. $2017 ; 17(265)$

39. Madi BC, Sandall J, Bennet R, et al. Effects of female relative support in labour: arandomized controlled trial. Birth. 1999;26:4-8.

40. Yuenyong S, O’Brein B, Jirapeet V. Effects of labour support from close female relative on labour and maternal satisfaction in a Thai setting. $J$ Obst Gynecol Neonatal Nurs. 2012; 41:45-56.

41. Maimbolwa MC, Sikazwe N, Yamba B. Views on involving a social support person during labour in Zambian maternities. J Midwifery Women's Health. 2001;46:226-234. 